\title{
Mediciones enredadas. La importancia de los indicadores mediático-culturales para el análisis de los sistemas mediáticos
}

\author{
Ruth Ainhoa DE Frutos García \\ Universidad de Málaga \\ ruth.defrutos@uma.es
}

\begin{abstract}
Resumen:
Este artículo plantea las bases para la realización de una investigación sobre los indicadores mediático-culturales, instrumentos imprescindibles para el análisis de los entornos mediáticos en la actualidad. La pertinencia de este estudio se basa en las nuevas características de los sistemas mediáticos, determinadas por la homologación cultural generada por la globalización pero también por las sinergias propias de instrumentos creados por entidades internacionales que son sometidas a diversas tensiones. Por tanto, resulta imprescindible conocer la naturaleza de las herramientas de medición de los entornos mediáticos para saber discernir si el análisis que se realiza a través de ellos es eficaz.
\end{abstract}

Palabras clave: Sistema mediático; indicadores de desarrollo mediático; estudios comparados

\section{Measurements tangled. The Importance of Media-Cultural Indicators for the Analysis of Media Systems}

\begin{abstract}
:
This paper presents the basis for conducting an investigation into the media-cultural indicators, essential instruments for the analysis of media environments today. The relevance of this study is based on the new features in media systems, determined by cultural approval generated by globalization but also by the characteristics of instruments created by international entities which are subjected to various stresses synergies. Therefore, it is essential to know the nature of the measurement tools of media environments to discern whether the analysis is performed through them is effective.
\end{abstract}

Key Words: Media System; Media Development Indicators; Comparative Studies

\section{Referencia normalizada:}

Frutos García, R. A. de (2014): Mediciones enredadas. La importancia de los indicadores mediático-culturales para el análisis de los sistemas mediáticos. Historia y Comunicación Social. Vol. 19. Núm. Especial Marzo. Págs. 525-537.

Sumario: 1. Introducción. 1.1. Pertinencia y objeto de estudio. 2. Globalización, democracia degradada e indicadores mediático-culturales 3. Hipótesis, objetivos y método dela investigación 3.1. Hipótesis 3.2. Objetivos 3.2.2. Objetivos específicos 3.3. Pluralidad metodológica acumulativa 4. Futuros resultados 


\section{Introducción}

Entre las numerosas cuestiones asociadas a la evaluación de los sistemas mediáticos se haya la de los procedimientos utilizados o potencialmente utilizables para dimensionar la envergadura de los diferentes entornos y sus características básicas: libertad de expresión, transparencia, género, alfabetización mediática, interacción con la audiencia, etc. Teniendo en cuenta este escenario, la investigación que se presenta en las siguientes páginas se plantea las siguientes preguntas: ¿Cómo es posible medir el grado de desarrollo mediático logrado por un determinado sistema mediático? ¿Son suficientemente satisfactorios los criterios y procedimientos que actualmente se utilizan para evaluar dicho entorno?

Este trabajo pretende eludir debates terminológicos sobre el término desarrollo ${ }^{1}$ por lo que, en adelante, se hablará de indicadores mediático-culturales. Según el Diccionario de la Real Academia de la Lengua Española, esta definición se concretaría en "que indica o sirve para indicar", mientras que el apellido mediático-cultural aproxima el término al sector de los medios de comunicación y a su importancia como instrumentos culturales. Esta apreciación ha dado lugar a diversas interpretaciones a lo largo de la historia.

A nivel internacional la Organización de Naciones Unidas para la Educación, la Ciencia y la Cultura (UNESCO) denomina a estos instrumentos indicadores de desarrollo mediático y los define como "herramientas diagnósticas antes que mandamientos conceptualizados con el propósito de ayudar con los programas de desarrollo mediático" (UNESCO, 2008: 5).

Estos instrumentos de medición son especialmente controvertidos debido a que no siempre responden a las necesidades reales de los análisis comparados, especialmente cuando no recogen adecuadamente las características propias de los sistemas mediáticos analizados.

No obstante, es necesario destacar la importancia de los indicadores mediático-culturales para entender la realidad, como un vehículo para ordenar la información y simplificar su conocimiento, generando formas más sencillas, aunque no simples, de comprender el mundo complejo (Stevens y Espeland, 2005). Desde esta perspectiva, el entendimiento de la realidad mediática de un determinado sistema respalda la construcción de políticas públicas de comunicación eficaces, que permitan la creación escenarios mediáticos de regeneración democrática y empoderamiento de la ciudadanía (Giannone, 2010). Así, estas herramientas de medición están llamadas a evolucionar en la dirección de la transparencia, la igualdad de género y la promoción de la democracia y la cultura de paz, como valores determinantes de un nuevo paradigma social (Nosty, 2011).

Se constituyen, por tanto, como una herramienta neutra y objetiva para analizar en un determinado sistema los aspectos de la ecología mediática y así diagnosticar, de una manera rigurosa, el estado de la comunicación en un determinado contexto mediático. Sin embargo, han de aplicarse teniendo en cuenta el contexto social, 
político, profesional y económico de cada país (UNESCO, 2008: vii). Precisamente dicho contexto puede llegar a condicionar la naturaleza y la objetividad de dichos instrumentos de medición de los entornos mediáticos. Para Diego Giannone:

"El proceso de medición se produce dentro de un contexto histórico y político que condiciona inevitablemente tanto al observador que elabora el instrumento de medición que la elección de la teoría que subyace de la propia medición" (2013, en prensa).

El análisis de los sistemas mediáticos a través de indicadores mediático-culturales han conformado una línea de investigación específica en la historia de los estudios de Comunicación (Nosty²; Vidal Beneyto, 2006), especialmente vinculada a los Estudios Culturales y a la Economía Política de la Comunicación (Marqués de Melo, 2012; Bolaño, Mastrini y Sierra, 2005).

Pese a que empiezan a consolidarse estudios que analizan el comportamiento de los consumos mediático-culturales ${ }^{3}$, permitiendo realizar estudios comparados y series históricas, existen otra serie de instrumentos que no promueven dichas evaluaciones, ya sea porque no persiguen dicho objetivo, como pueden ser los indicadores de desarrollo mediático de UNESCO (2008); o porque su metodología les impide realizar series históricas, como los indicadores de libertad de expresión de Freedom House ${ }^{4}$.

Por tanto, los resultados de la tesis doctoral ${ }^{5}$ que se presentan en este texto tiene como propósito paliar las carencias sobre el conocimiento real de los entornos mediáticos a través del abordaje de la importancia de los indicadores mediático-culturales, permitiendo evaluar de forma eficaz dichos sistemas mediáticos y, por ende, generando políticas de comunicación eficientes.

Derivado de la premisa anterior surge el principal objetivo del trabajo, que consiste en detectar los puntos débiles y fuertes de los sistemas de indicadores mediático-culturales existentes, con el fin de ahondar en la importancia de la creación de un nuevo corpus que supere las debilidades y optimice las fortalezas de dicho corpus, permitiendo la realización de estudios comparados y series temporales.

Las singularidades de los indicadores mediático-culturales son fácilmente identificables por personas que han participado en su definición, aplicación o análisis crítico; al ser las que de una forma u otra han puesto en funcionamiento el engranaje de la medición mediática a través de dichos instrumentos. Por este motivo, el estudio empírico tendrá como referencia las opiniones de un panel de expertos diseñado para tal efecto y que permitirá evaluar la eficacia de seis familias de indicadores: aquellos que hacen referencia a la implicación de las audiencias como indicador del desarrollo de un determinado entorno, pero también el grado de libertad de expresión, el papel de la mujer en los medios de comunicación, el grado de transparencia de los mismos y la alfabetización mediática que aportan a la sociedad.

El análisis comparado de las características de los indicadores mediático-culturales objeto de estudio no sólo es una estrategia adecuada para evaluar una realidad 
mediática de una determinada zona, sino una forma de asignar un valor relativo a distintos escenarios, pudiendo generar políticas públicas de comunicación eficientes.

\subsection{Pertinencia y objeto de estudio}

El análisis de los diferentes entornos mediáticos a través de baremos objetivables y, por tanto, comparables en diversos escenarios mediáticos ha sido la máxima que han intentado alcanzar diversas organizaciones internacionales y académicos de todo el mundo (Hallin y Mancini, 2005). El campo comunicacional no ha sido el único que ha intentado promover este tipo de estudios comparados sino que, de forma general, todas las áreas de conocimiento ${ }^{6}$ han experimentado un proceso de uniformización $u$ homologación para alcanzar dicho fin, directamente relacionado con el mundo globalizado en el que vivimos.

Sin embargo, los estudios al uso no ofrecen una perspectiva integradora, es decir, un análisis sistemático del espacio mediático en su conjunto, por lo que, desde esa visión fragmentaria de partida, es difícil evaluar el papel que, en cada país, juega cada uno de los medios, el tiempo de uso social, la credibilidad e influencia que alcanzan y su incidencia en la sedimentación de valores cívicos y culturales (Prince, Abbott y Morgan, 2011; Tolilla, 2010; Díaz Nosty, 2005).

Por otra parte, la investigación que se presenta puede ser de utilidad no sólo para la comunidad académica dentro de la rama de la Comunicación y del Periodismo, sino a todos aquellos organismos internacionales que realmente pretendan que sus indicadores sean eficientes para realizar análisis comparados de los diversos entornos mediáticos.

En conclusión, un análisis crítico de los datos derivados de este trabajo generará una ventana de oportunidad a la investigación española en el ámbito internacional y, por otra parte, una tendencia de futuro clara al estudiar los nuevos escenarios que se plantearán en esta cuestión tan importante para el desarrollo democrático de un país como la medición del sistema mediático.

\section{Globalización, democracia degradada e indicadores mediático-culturales}

Las peculiaridades del mundo global acrecientan la importancia de la medición y el análisis de los diversos sistemas mediáticos. Por ello, esta investigación aborda las pautas de evaluación que son utilizadas por los diversos indicadores mediático-culturales de distintas organizaciones ${ }^{7}$.

Por otro lado, debido a las profundas transformaciones que el proceso de modernización tecnológica impone en el sector mediático actual (Norris, 2012: 56) es fundamental analizar, reinterpretar esa realidad y visibilizar las modificaciones que se producen a través de instrumentos que puedan respaldar estos objetivos. 
A lo largo del trabajo se esgrime el término de instituciones de comunicación o medios, entendidos como el conjunto de organizaciones que utiliza tecnologías específicas para realizar una comunicación entre seres humanos, esto es, un grupo de emisoras d radio, de televisión (abierta o de pago), periódicos, revistas, cine y otras instituciones que hacen uso de recursos tecnológicos en la llamada comunicación de masa (Lima, 2006; Oliveira Paulino, 2009: 12).

La primera parte del estudio aporta un marco teórico al significado de los indicadores mediático-culturales como instrumento para generar políticas públicas de comunicación con una finalidad clara: la protección de los derechos y libertades de la ciudadanía (De Morães, 2011; Torrico, 2009). De hecho, la inclusión a los derechos de la comunicación en las políticas públicas ha adquirido un alcance tanto nacional como supranacional; fenómeno que ha estado sometido a presiones de todo tipo: gubernamentales, empresariales, religiosas, individuales, etc. Fruto de dichas presiones, se ha generado lo que Díaz Nosty (1995) describe como democracia degradada y que permite contextualizar en el tejido globalizado en el que vivimos, la importancia de la medición de los entornos mediáticos a través de los pespuntes creados por indicadores eficientes.

Ignacio Ramonet fue el primer autor que habló de la creación de un "quinto poder", un nuevo núcleo de poder, cuyo protagonista sería la sociedad civil, orientado a "denunciar el superpoder de los medios de comunicación, de los grandes grupos mediáticos, cómplices y difusores de la globalización liberal" (Ramonet, 2003, online; ídem, 2004). Para el director de la redacción de Le Monde Diplomatique en castellano es importante disponer finalmente de un arma para dicho fin y, precisamente, dicho arma necesita unos indicadores o índices que permitan cuantificar cuál es el estado del sector mediático en un determinado sistema. Sin embargo, más allá del valor de dichos instrumentos de medición en abstracto, la eficacia de los mismos deriva de su correcta aplicación, motivada por el carácter de las organizaciones que promueven dichos indicadores, así como de su propia finalidad.

En este sentido, se pueden aplicar los grandes desafíos que Herschmann, dos Santos y Albornoz identifican para los observatorios de comunicación en los propios indicadores mediático-culturales:

a) as debilidades na coordenação interna e carência de uma integração mais intensa e efetiva com outros observatorios e instituções,

b) risco de ser identificado pelo público apenas como parte da engranegem burocrática estatal,

c) fragilidade dos canais de diálogo com os cidadãos e risco da adoção de uma postura tecnocrática,

d) risco de ser constituir em instancia de lobby dos poderes políticos e económicos. (Albornoz, dos Santos y Herschmann, 2008: 11) 
Son precisamente estos desafíos los que generan la idea de componer un bloque en la investigación destinada a la revisión teórico-metodológica de los principales indicadores mediático-culturales utilizados a nivel internacional, observando sus características intrínsecas y extrínsecas previas al auténtico estudio empírico.

\section{Hipótesis, objetivos y método de la investigaión}

\subsection{Hipótesis}

Este trabajo parte de dos hipótesis principales y de una serie de hipótesis secundarias que se derivan de las primeras.

- Los principales corpus de indicadores mediático-culturales existentes en la actualidad no siempre responden a las necesidades reales de los análisis comparados, especialmente cuando no recogen eficazmente las características propias de los entornos mediáticos.

- Los indicadores mediático-culturales están llamados a evolucionar en la dirección de la transparencia, la igualdad de género la democracia y la cultura de paz, como valores determinantes de un nuevo paradigma social.

Relacionadas con estas hipótesis, se plantean las siguientes hipótesis secundarias:

- Los indicadores mediático-culturales aplicados en la actualidad son creados por entes con claros intereses políticos, económicos, sociales o religiosos, lo que podría dificultar su correcta aplicación y/o contaminación de los resultados.

- Los indicadores mediático-culturales existentes son parciales, es decir, hacen referencia solo a un aspecto del entorno mediático como puede ser la transparencia, el género, la alfabetización mediática, la implicación de las audiencias o la libertad de expresión, sin que puedan ser aglutinados en un corpus único.

- Los indicadores mediático-culturales se están convirtiendo en un instrumento de proyección pública para algunos organismos que pretenden consolidar su imagen mediante el esfuerzo de creación de estos instrumentos. Sin embargo, este tipo de herramientas se muestran en ocasiones ineficaces si no pueden ser fuentes de análisis comparados entre diversos entornos mediáticos.

- Los indicadores mediático-culturales promovidos por organizaciones públicas nacionales o internacionales muestran más deficiencias específicas debido a la presión política.

- En el caso de producirse presiones en organismos internacionales, podrían estar directamente relacionadas con el posicionamiento político de los estados miembros, pero también con el afán de éstos por la ocultación de su infor- 
mación o, si cabe, la escasa voluntad de transparencia sobre la información referente a sus entornos mediáticos.

- En línea con el desiderátum de transparencia, y a tenor de los actuales desarrollos tecnológicos y de las prácticas sociales, la evolución de los sistemas mediático-culturales está estrechamente relacionada con la participación social y el derecho de acceso a los medios de la ciudadanía.

Además de la verificación de las hipótesis que se plantean en el anterior epígrafe, esta investigación pretende generar una herramienta estratégica de medición eficaz de los distintos entornos mediáticos. Para conseguir dicha meta, se fijan los siguientes objetivos.

\subsection{Objetivos}

El objetivo principal es el de conocer las características de los principales corpus de indicadores mediático-culturales existentes para poder evaluar si estos sirven para el desarrollo efectivo de estudios comparados entre diversos entornos mediáticos y líneas temporales y conocer así cuál es el nivel de eficacia de los corpus de indicadores existentes.

En este sentido, el primer objetivo sería el de analizar críticamente los principales sistemas de indicadores mediático-culturales que existen en la actualidad ${ }^{8}$, a través del estudio pormenorizado de aquellos que por su relevancia y especialización están directamente relacionados con el presente objeto de estudio.

Para ello, se estudiarán las posturas de los expertos que han trabajado en su definición, aplicación y análisis crítico así como se realizará una evaluación de los corpus de indicadores a través de fuentes secundarias. De esta manera, se constatará si existen deficiencias en dichas herramientas de medición y, de ser así, las posibilidades de perfeccionamiento.

Otro de los objetivos planteados será el de evaluar si los indicadores actuales permiten realizar análisis temporales y comparados entre distintos entornos mediáticos, lo que permitiría general políticas públicas de comunicación más eficaces.

\subsubsection{Objetivos específicos}

Entre los objetivos específicos de la investigación se encuentran:

- Fomentar el debate sobre la construcción de sistemas de medición cuantitativos y cualitativos de los entornos mediático-culturales. Para ello, seleccionaremos las opiniones de expertos internacionales que han trabajado en la definición y aplicación de distintos corpus de indicadores mediáticos evaluaremos las principales características metodológicas de los métodos de medición de los sistemas mediáticos. Asimismo, podremos comprobar la importancia del diseño multimétodo para el análisis de los diferentes entornos mediáticos en el mundo. 
- Realizar un análisis comparado de los principales corpus de indicadores mediático-culturales a través de las opiniones de los especialistas que han participado activamente en la realización de los mismos de una forma teórica o práctica.

- Determinar las estrategias de mejora del conjunto de medidas analizadas a partir de los resultados obtenidos a través de análisis críticos desarrollados previamente. Se trata de sentar las bases para la mejora sistemática de los corpus de indicadores actuales para que se puedan desarrollar series temporales y estudios comparados.

- Identificar los nuevos escenarios estratégicos en los que se podrían proyectar los indicadores mediático-culturales resultantes del estudio. En este sentido, se propondrá la aplicación de los conjuntos de indicadores mediático-culturales en tres escenarios diferentes (positivo, negativo y neutral) para evaluar su grado de actuación en cada caso.

- Sentar las bases de futuras investigaciones sobre el desarrollo y la evolución de indicadores mediático-culturales.

\subsection{Pluralidad metodológica acumulativa}

Frente a la ausencia de investigaciones previas sobre el objeto de estudio es necesaria la aplicación de un diseño metodológico complejo, que sea capaz de evaluar la naturaleza de los indicadores de forma estructurada, por lo que se ha optado por la aplicación de herramientas cuantitativas y cualitativas combinando métodos, filosofía y orientaciones de diseño metodológico mixto (Creswell, 2012; Creswell y Plano Clark, 2011).

Como se ha propuesto anteriormente, en la presente investigación se procura evaluar las características de dichos indicadores a través de los expertos que los han identificado o aplicado, conociendo la valoración que hacen de ellos y generando estrategias de mejora, si fuese necesario (Manson, 2006: 3).

Por tanto, el diseño multimétodo aplicado en la presente investigación, determinado por la pluralidad metodológica acumulativa, está basado en la ejecución secuencial de tres métodos diferentes, tras una fase preliminar de revisión bibliográfica:

- En una primera fase se plantea un método Delphi, por el cual especialistas del ámbito de la comunicación y/o miembros de las comisiones que han participado en la definición, aplicación o análisis crítico de alguno de los corpus de indicadores mediático-culturales estudiados darán su parecer sobre los factores positivos y negativos de los mismos.

- En una segunda fase se aplica la matriz DAFO a las conclusiones obtenidas de las opiniones de los expertos en la primera fase. En este sentido, el objetivo de esta segunda fase metodológica es el estudio pormenorizado de las principales debilidades, amenazas, fortalezas y oportunidades de cada uno de los 
corpus de los indicadores mediático-culturales analizados, con el fin de crear estrategias de eliminación de los puntos deficitarios y promoción de aquellos elementos más eficaces.

- En una tercera fase, la investigación se basa en un análisis estratégico de la situación de los indicadores mediático-culturales en la actualidad, así como el diseño de escenarios tendenciales. Asimismo, se plantea la posibilidad de cuatro escenarios que permitan observar el funcionamiento de los indicadores en diversas situaciones.

\section{Futuros resultados}

Por todo ello, la tesis doctoral que se ha presentado resumidamente en este pretende verificar o refutar las hipótesis enumeradas a través de una investigación que, de ser cierto el planeamiento expuesto, permitirá desvelar si realmente los indicadores mediático-culturales actuales permiten realizar series temporales y estudios comparados entre diferentes entornos mediáticos, las razones por las que estos instrumentos tienen tanta importancia en la realidad y, de ser deficitarios, qué mecanismos de mejora se podrían aplicar a los corpus de indicadores mediático-culturales analizados para promover políticas públicas de comunicación eficaces.

\section{Bibliografía}

ALBORNOZ, L. A., HERSCHMANN, M. y SANTOS, S. (2008). Analisando o crescimento do número de observatorios de comunicação, cultura e informação no Brasil. En XVII Encontro da Compós. San Paulo. Disponible en: http://www2. eptic.com.br/sgw/data/bib/artigos/20c36452adba687023fbd7bdd1b846c4.pdf. [30-07-2013].

BERICAT, E. (1998). La integración de los métodos cuantitativo y cualitativo en la investigación social. Barcelona: Ariel.

CRESWELL, J. (2012). Qualitative Inquiry and Research Design: Choosing Among Five Approaches. Thousand Oaks: CA. Sage.

BOLAÑO, C.; MASTRINI, G. y SIERRA, F. (2005). Economía política, comunicación y conocimiento: una perspectiva crítica latinoamericana. Sevilla/Buenos Aires: Junta de Andalucía/La Crujía Ediciones.

CALLEJO, J. y VIEDMA. A. (2006). Proyectos y estrategias de Investigación Social: la perspectiva de la intervención. Madrid: McGraw Hill.

CRESWELL, J. y PLANO CLARK, V.. (2011). Designing and conducting mixed methods research. Thousand Oaks: CA. Sage.

DE MORAES, D. (2011). Voces Abertas da América Latina. Estado, políticas públicas e democratização da comunicação. Rio de Janeiro: Mauad X y Faperi. 
DÍAZ NOSTY, B. (2011). El libro negro del Periodismo. Madrid: Asociación de la prensa de Madrid y Cátedra UNESCO de Comunicación Universidad de Málaga.

DÍAZ NOSTY, B. (2005). El déficit mediático. Donde España no converge con Europa. Barcelona: Editorial Bosch.

DÍAZ NOSTY, B. (1995): Los medios y la hipótesis de la democracia degradada. Málaga: Ser. Pub. UMA.

FREEDOM HOUSE (2012). Freedom of the Press. Methodology. www.freedomhouse.org.

GIANNONE, D. (2010). "Political and ideological aspects in the measurement of democracy: the Freedom House case". En Democratization, ${ }^{\circ}$ 17:1. p. 68-97. Disponible en: http://dx.doi.org/10.1080/13510340903453716. [29-09-2013].

KRIPPENDORFF, K. (2004). Content Analysis. An Introduction to ist Methodology. Thousand Oack, CA. Sage Publish.

LANDMAN, T. y HÄUSERMANN, J. (2003). Map-Making and Analysis of the Main International Iniciatives on Depeloping Indicators on Democracy and Good Governance. Final Report. Essex: University of Essex - Human Rights Center.

LAZCANO PEÑA, D. (2009). "Consideraciones epistemológicas en torno a la comunicación y los medios". En SANTANDER MOLINA, P. (2009). Analizando los medios y la comunicación: teoría y métodos. Ediciones Universitarias de Valparaiso. Valparaíso.

LIMA, V. A. (2006). Mídia: crise política e poder no Brasil. São Paulo: Fundación Perseu Abramo.

MASON, J. (2006). "Real Life Methods Working Papers: Six strategies for mixing methods and linking data". En: ESRC National Centre for Research Methods. NCRM Working Paper Series 4/6. University of Manchester: Manchester. Disponible en: http://www.reallifemethods.ac.uk/publications/workingpapers/2006-07-rlm-mason.pdf. [29-03-2013].

MUNCK, G. L. (2006). "Monitoreando la democracia: Profundizando un consenso emergente". En: Revista de ciencia política. . . 158-168. Disponible en: http://www. scielo.cl/scielo.php?script $=$ sci_arttext\&pid $=$ S0718-090X2006000100010\&ln$\mathrm{g}=$ es\&tlng=es. 10.4067/S0718-090X2006000100010[4-08-2013].

NORRIS, P, (2012). "Political mobilization and social networks. The example of the Arab spring". En KERSTING, N (Dir.) (2012). Electronic Democracy. The Development of the Discipline Book Series. Budrich. LeverkusenLANDETA, J. (1999). El método Delphi. Barcelona: Ariel.

OLIVEIRA PAULINO, F. (2009). Responsabilidade Social da Mídia. Análise conceitual e perspectivas de aplicação. Brasília: Casa das Musas.

PRICE, M. E.; ABBOTT, S. y MORGAN, L. (Eds.) (2011). Measures of Press Freedom and Media Contributions to Development. Evaluating the Evaluators. Oxford: Mass Communication and Journalism..

RAMONET, I. (2004). "Información, comunicación y globalización, El quinto poder". En: Revista Latinoamericana de Comunicación CHASQUI. Centro Internacional de Estudios Superiores de Comunicación para América Latina. Quito. p. 26-30. 
RAMONET, I. (2003). "El quinto poder". En: Le Monde Diplomatique. Disponible en: http://www.lemondediplomatique.cl/El-quinto-poder.html. [30-08-2013].

SANDELOWSKI, M., VOILS, C. y KNAFL, G. (2009). "On quantitizing”. En: Journal of Mixed Methods Research, $\mathrm{n}^{\circ}$ 3, vol 3. p. 208-222.

SEN, A. (2000). Desarrollo y libertad. Madrid: Planeta.

STEVENS, M. y ESPELAND. W. (2005). "Commensuration". En: Encyclopedia of Social Measurement. Elsevier Academic Press: Amsterdam. p. 375-378.

TOLILA, P (2010). "¿Qué es un indicador? Problemática general, historia, usos y límites". E:n Consejo Nacional para la Cultura y las Artes de México (CONALCULTA). Seminario construcción de indicadores de desarrollo cultural comunitaria. Ciudad de México. Disponible en: http://issuu.com/flyoscar/docs/libro. [27-12-2012].

TORRICO, E. (2009). "Periodismo, ética y democracia". Quito, 20-22 de octubre de 2009. Disponible en: http://es.scribd.com/doc/26685844/Ponencia-CIESPAL-Erick-Torrico. [29-07-2013].

TORRICO, E. (2004). Abordajes y Periodos de la Teoría de la Comunicación. Buenos Aires: Grupo Editorial Norma.

UNESCO (2008), Indicadores de Desarrollo Mediático: Marco para evaluar el desarrollo de los medios de comunicación social. Paris: UNESCO.

VERD, J.M. y LÓPEZ, P. (2008). "La eficiencia teórica y metodológica de los diseños multimétodo". En: Empiria, Revista de Metodología de Ciencias Sociales, $\mathrm{n}^{\mathrm{o}}$ 16, vol 2. p. 13-45. Disponible en: http://www.hugoperezidiart.com.ar/tallerdetesis-pdf/103-verd-lopez.pdf. [28-03-2013].

VIDAL BENEYTO, José y DÍAZ BARRADO, C. (Coord.) (2008). América Latina, hacia su unidad. Modelos de integración y procesos integradores. Valencia: Amela y Ed. Pre-Textos.

PROGRAMA DE NACIONES UNIDAS PARA EL DESARROLLO (PNUD) (1990). Informe Sobre Desarrollo Humano. Bogotá: Panamericana Formas e Impresos.

\section{Notas}

1 La idea preliminar de "desarrollo", según aportaciones de diferentes autores, fue expresada por el presidente de Estados Unidos Woodrow Wilson en 1918 en su Discurso de los catorce puntos. Sin embargo, el contexto geopolítico de la descolonización, el Plan Marshall y otros fenómenos del s. XX generalizaron el concepto. Entre las teorías clásicas del desarrollo se encuentran las de la modernización, el tercer mundo y la dependencia pero la presente investigación abordará el término desarrollo desde una perspectiva humana, en la que dicho concepto no sé vinculará simplemente al progreso industrial de un determinado entorno sino también al enfoque social, cultural y ambiental. En este contexto, el desarrollo se identifica también con el progreso respecto a los medios de comunicación: transparencia, libertad de expresión, inclusión de la perspectiva de género en los medios, alfabetización mediática e interacción de las audiencias. Por tanto, cuando se hable de desarrollo en estas páginas se contemplarán las definición de desarrollo como "aumento de las opciones para que las personas puedan mejorar su vida", propuesta por primera vez en el Informe sobre desarrollo humano del PNUD (1990: 33), y desarrollo humano "como el proceso de expansión de las libertades que goza el pueblo" (Sen, 2000: 13). 
2 El profesor de la Universidad de Málaga dirigió, entre 1989 y 1996, los informes Comunicación Social/Tendencias de la Fundación FUNDESCO; entre 1997 y 2001, el Informe Anual de la Comunicación (Eds. Zeta, Barcelona); el Informe Anual de la Profesión Periodística (2004); los informes La UE en los medios de Comunicación (1994-1997), y los informes de medios (España y América Latina) de 2006 y 2007 (Ariel, Barcelona/ Fundación Telefónica). Asimismo es autor del análisis comparado de medios en Europa El déficit mediático: Donde España no converge con Europa (Bosch, 2005), y Libro negro del periodismo en España (2011).

3 Por citar un ejemplo, la Oficina Europea de Estadísticas (Eurostat), publica desde 2007 un informe anual titulado Estadísticas culturales de la UE, disponible en inglés, francés y alemán. Este informe pone de relieve la importancia mayor y el reconocimiento del papel que juega la cultura en la cohesión social, intentando clarificar los enlaces que existen entre la cultura y el desarrollo social y económico, apoyándose en datos del Eurobarómetro, el Sistema Estadístico Europeo, informes de UNESCO, etc.

4 Si bien Freedom House alude a que su índice permite comparar un sistema mediático durante todo el arco de tiempo analizado por la organización (Freedom House, 2012), los cambios en la composición interna del indicador de FH impiden la realización de series históricas eficaces con el fin de analizar los distintos sistemas mediáticos a lo largo del tiempo. En 1994 se introdujo una escala de 100 puntos, segregando los países por libre, parcialmente libre y no libre. Sin embargo, hasta 1988 el instrumento daba información independiente de la prensa y de la radio y la televisión y, un año después, aparecen como un único dato. (Para más información sobre el análisis cualitativo del contenido del índice de FH ver Giannone, 2010; Krippendorff, 2004; Landman y Hâusermann, 2003).

5 Este artículo es una síntesis del proyecto de tesis doctoral de la autora que ha sido financiado a través de una beca de Formación de Profesorado Universitario (FPU).

6 Según Munck, después de la Guerra Fría la preocupación por el estado de la democracia generó numerosas iniciativas, que continúan hoy en día, para monitorear distintas esferas relacionadas directamente con la calidad de la vida democrática en los diversos países: "El monitoreo de la democracia se concentró inicialmente en la observación electoral. (...) Sin embargo, rápidamente el alcance de las actividades de monitoreo se expandió más allá de las elecciones y comenzó a incluir un conjunto de temáticas directa o estrechamente relacionadas con la democracia, tales como las fuentes de financiamiento de los partidos políticos, el acceso público a la información y la libertad de prensa, la corrupción de funcionarios públicos, el funcionamiento del poder judicial, el respeto a los derechos humanos, y la implementación de políticas públicas. (...)” (Munck, 2006: 158).

7 En este sentido, el presidente del Consejo Intergubernamental del programa Internacional para el Desarrollo (PIDC) en 2008 Walter Fust ya explicaba que la realización de un marco para evaluar el desarrollo mediático "mejora los esfuerzos por el desarrollo mediático de dos maneras: desde el principio, permitiendo una mejor distribución del apoyo al desarrollo de medios mediante la identificación sistémica de las necesidades del sector mediático de un determinado país; y hasta el final, facilitando la medición del impacto de las intervenciones en el campo" (Fust, citado por UNESCO. 2008: vii).

8 Dada la ingente cantidad de instrumentos de esta naturaleza existentes en la actualidad, el estudio analiza los indicadores mediático-culturales de seis ámbitos determinados (alfabetización mediática, libertad de expresión, género en medios de comunicación, transparencia y participación de la audiencia). 


\section{La autora}

Ruth de Frutos es becaria FPU del Departamento de Periodismo de la Universidad de Málaga desde mayo 2011, donde trabaja en su tesis doctoral sobre indicadores mediático-culturales y es miembro del proyecto del Plan Nacional I+D +i "Comunicación y Cambio Climático: Estrategias de Traslación Mediática del Consenso Científico a la Opinión Pública" (CSO2010-19725).

Licenciada en Periodismo por la Universidad de Valladolid, donde simultaneó sus estudios con los de Derecho, ha trabajado en la Cátedra UNESCO de Comunicación de la Universidad de Málaga (2009-2010) y en el Centro UNESCO de Turín (20102011). Anteriormente, ha trabajado en medios y varias empresas de comunicación como El Norte de Castilla, Nuevo cuño o la agencia de noticias EFE.

$\mathrm{Su}$ interés por los derechos humanos la ha llevado a colaborar con la Asamblea Permanente de los Derechos Humanos (Argentina) dentro del Programa de Prácticas universitarias en el Ámbito de la Cooperación Internacional para el Desarrollo (PACID) y ser responsable del gabinete de comunicación de Amnistía Internacional en Valladolid, Granada y Málaga.

Actualmente, combina sus funciones como investigadora de la Universidad de Málaga con las de miembro del Centro de Cooperación Internacional para el Desarrollo de la Universidad de Valladolid. 